\title{
A Novel Fifth-Degree Cubature Kalman Filter for Real-Time Orbit Determination by Radar
}

\author{
Zhaoming Li, ${ }^{1}$ Wenge Yang, ${ }^{2}$ Dan Ding, ${ }^{2}$ and Yurong Liao ${ }^{2}$ \\ ${ }^{1}$ Graduate School, Academy of Equipment, Beijing 101416, China \\ ${ }^{2}$ Department of Optical and Electrical Equipment, Academy of Equipment, Beijing 101416, China \\ Correspondence should be addressed to Zhaoming Li; lizhaomingzbxy@163.com
}

Received 26 February 2017; Accepted 29 May 2017; Published 24 August 2017

Academic Editor: Huanqing Wang

Copyright (c) 2017 Zhaoming Li et al. This is an open access article distributed under the Creative Commons Attribution License, which permits unrestricted use, distribution, and reproduction in any medium, provided the original work is properly cited.

\begin{abstract}
A novel fifth-degree cubature Kalman filter is proposed to improve the accuracy of real-time orbit determination by ground-based radar. A fully symmetric cubature rule, approaching the Gaussian weighted integral of a nonlinear function in general form, is introduced, and the specific points and weights are calculated by matching the monomials of degree not greater than five with the exact values. On the basis of the above rule, a novel fifth-degree cubature Kalman filter, which can achieve a higher accuracy than UKF and CKF, is derived under the Bayesian filtering framework. Then, to describe the nonlinear system more accurately, the orbital dynamics equation with J2 perturbation is used as the state equation, and the nonlinear relationship between the radar measurement elements and orbital states is built as the measurement equation. The simulation results show that, compared with the traditional third-degree algorithm, the proposed fifth-degree algorithm has a higher accuracy of orbit determination.
\end{abstract}

\section{Introduction}

With the increasing number of satellites launched into orbit every year, the monitoring and cataloguing of satellites play an important role in improving the rate of utilisation of space resources and alleviating the pressure on orbit resources. As a type of sensor in space surveillance systems, groundbased radar is equipped without considering the influence of the weather and other special circumstances, and the use of its measurement data for real-time orbit determination is a key technology in space target tracking $[1,2]$. Due to the nonlinearity of the satellite orbital dynamics model with the influence of orbital perturbation, the essence of orbit determination in real-time is to achieve the optimal estimation of the orbital state by means of nonlinear filtering technology under the Bayesian framework using the measured ranging, velocity, and angle data with measurement noise, which has important research value.

The core issue in nonlinear Kalman filtering is to calculate the intractable multidimensional vector integral such as the "nonlinear function $\times$ Gaussian probability density function (pdf)," for which it is difficult to achieve the analytical solution $[3,4]$. At present, two methods, including the approximation of the nonlinear function and the approximation of the Gaussian pdf, are mainly taken. In the former method, the nonlinear function is approximated by the polynomial and results in an extended Kalman filter (EKF) [5, 6], divided difference Kalman filter (DDKF) [7], and polynomial Kalman filter (PKF) [8, 9], where the first-order Taylor expansion, the multidimensional Stirling interpolation, and polynomials including Chebyshev and Fourier-Hermit are adopted to approximate the nonlinear function, respectively, in EKF, DDKF, and PKF. However, the aforementioned methods tend to be restricted when the system has strong nonlinearity with high dimensionality. For the latter, the Gaussian pdf is approximated using the deterministic sampling approach, which mainly includes the unscented transform (UT) and spherical-radial rule (SRR). Then, the unscented Kalman filter (UKF) $[10,11]$ and cubature Kalman filter (CKF) [12-14] are obtained by embedding UT and SRR into the Bayesian filtering framework, respectively, these have a wide range of applications in engineering [1520], but these two types of algorithm have only third-degree filtering accuracy, which is required to be further improved. 
In this paper, a novel fifth-degree cubature Kalman filter is proposed without differential operation to improve the filtering accuracy from third degree to fifth degree. The integral points with corresponding weights in the general cubature rule are calculated by matching the monomials of degree no more than five with their exact values in the fully symmetric region. Then, the proposed filtering method, which can achieve a higher accuracy compared to that with $\mathrm{UKF}$ and CKF, is deduced by embedding the novel fifthdegree cubature rule into the Bayesian filtering framework. The proposed filtering algorithm is applied in real-time orbit determination, and a more accurate orbit estimate is achieved.

The remainder of the paper is organised as follows: the traditional cubature Kalman filter is described in Section 2, the novel fifth-degree cubature rule and related Kalman filter are derived in Section 3, the mathematical models used for orbit determination are described in Section 4, the numerical experiment and results are presented in Section 5, and the conclusions are drawn in Section 6.

\section{The Traditional Cubature Kalman Filter}

The core problem in any nonlinear Kalman filter is to calculate the integral $\int_{\mathbf{R}^{n}} \mathbf{f}(\mathbf{x}) N\left(\mathbf{x} ; \overline{\mathbf{x}}, \mathbf{P}_{x}\right) d \mathbf{x}$, for which, in general, it is difficult to find the analytical solution, where $\mathbf{f}(\mathbf{x})$ denotes the arbitrary function, $N\left(\mathbf{x} ; \overline{\mathbf{x}}, \mathbf{P}_{x}\right)$ denotes the Gaussian pdf. Specifically, an integral of the form $I(\mathbf{f})=\int_{\mathbf{R}^{n}} \mathbf{f}(\mathbf{x}) e^{-\mathbf{x}^{\mathrm{T}} \mathbf{x}} d \mathbf{x}$ in the Cartesian coordinate system is considered. Let $\mathbf{x}=r \mathbf{y}$ with $\mathbf{y}^{\mathrm{T}} \mathbf{y}=1$, where $\mathbf{y}$ denotes the direction vector and $r \geq 0$ denotes the radius, so that $\mathbf{x}^{\mathrm{T}} \mathbf{x}=r^{2}$ and then the integral $I(\mathbf{f})$ can be rewritten in a spherical-radial coordinate system as follows:

$$
I(\mathbf{f})=\int_{0}^{\infty} \int_{U_{n}} \mathbf{f}(r \mathbf{y}) r^{n-1} e^{-r^{2}} d \sigma(\mathbf{y}) d r
$$

where $U_{n}$ is the surface of the sphere defined by $U_{n}=\{\mathbf{y} \in$ $\left.\mathbf{R}^{n}: \mathbf{y}^{\mathrm{T}} \mathbf{y}=1\right\}$ and $\sigma(\cdot)$ is the area element on $U_{n}$. Thus, the integral is decomposed into spherical integral $S(r)$ and radial integral $R$, respectively, and approximately represented using numerical integration as follows:

$$
\begin{aligned}
S(r) & =\int_{U_{n}} \mathbf{f}(r \mathbf{y}) d \sigma(\mathbf{y}) \approx \sum_{i=1}^{L_{s}} \omega_{s, i} \mathbf{f}\left(r \mathbf{y}_{i}\right) \\
R & =\int_{0}^{\infty} S(r) r^{n-1} e^{-r^{2}} d r \approx \sum_{j=1}^{L_{r}} \omega_{r, j} S\left(r_{j}\right),
\end{aligned}
$$

where $\left(\mathbf{y}_{i}, \omega_{s, i}\right)$ denote the integral points and weights of the spherical integral and $L_{s}$ denotes the number of integral points. Similarly, $\left(r_{j}, \omega_{r, j}\right)$ denote the integral points and weights of the radial integral, and $L_{r}$ denotes the number of points. From the third-degree spherical-radial cubature rule used in [12], we obtain that

$$
\begin{aligned}
S(r) & =\frac{A_{n}}{2 n} \sum_{i=1}^{n}\left[\mathbf{f}\left(r \boldsymbol{\xi}_{i}\right)+\mathbf{f}\left(-r \boldsymbol{\xi}_{i}\right)\right] \\
R & =\frac{1}{2} \Gamma\left(\frac{n}{2}\right) S\left(\sqrt{\frac{n}{2}}\right),
\end{aligned}
$$

where $A_{n}=2 \sqrt{\pi^{n}} / \Gamma(n / 2)$ is the surface area of the unit sphere, $\Gamma(z)=\int_{0}^{\infty} e^{-t} t^{z-1} d t$ is the Gamma function, and $\xi_{i}$ denotes the unit vector with the $i$ th element being $1 . S(r)$ is substituted into $R$, to get

$$
\begin{aligned}
I(\mathbf{f}) & =\sum_{j=1}^{L_{r}} \sum_{i=1}^{L_{s}} \omega_{r, j} \omega_{s, i} \mathbf{f}\left(r_{j} \mathbf{y}_{i}\right) \\
& =\sum_{j=1}^{L} \frac{1}{2} \Gamma\left(\frac{n}{2}\right) \sum_{i=1}^{n} \frac{A_{n}}{2 n}\left[\mathbf{f}\left(r_{j} \boldsymbol{\xi}_{i}\right)+\mathbf{f}\left(-r_{j} \boldsymbol{\xi}_{i}\right)\right] \\
& =\frac{\sqrt{\pi^{n}}}{2 n} \sum_{i=1}^{n}\left[\mathbf{f}\left(\sqrt{\frac{n}{2}} \boldsymbol{\xi}_{i}\right)+\mathbf{f}\left(-\sqrt{\frac{n}{2}} \boldsymbol{\xi}_{i}\right)\right],
\end{aligned}
$$

Due to identity equation

$$
\begin{aligned}
& \int_{\mathbf{R}^{n}} \mathbf{f}(\mathbf{x}) N\left(\mathbf{x} ; \overline{\mathbf{x}}, \mathbf{P}_{x}\right) d \mathbf{x} \\
& \quad=\frac{1}{\sqrt{\pi^{n}}} \int_{\mathbf{R}^{n}} \mathbf{f}\left(\sqrt{2 \mathbf{P}_{x}} \mathbf{x}+\overline{\mathbf{x}}\right) e^{-\mathbf{x}^{\mathrm{T}} \mathbf{x}} d \mathbf{x},
\end{aligned}
$$

it may be seen that

$$
\begin{aligned}
& \int_{\mathbf{R}^{n}} \mathbf{f}(\mathbf{x}) N\left(\mathbf{x} ; \overline{\mathbf{x}}, \mathbf{P}_{x}\right) d \mathbf{x} \\
& \quad=\frac{1}{2 n} \sum_{i=1}^{n}\left[\mathbf{f}\left(\sqrt{n \mathbf{P}_{x}} \xi_{i}+\overline{\mathbf{x}}\right)+\mathbf{f}\left(-\sqrt{n \mathbf{P}_{x}} \xi_{i}+\overline{\mathbf{x}}\right)\right] .
\end{aligned}
$$

The calculation process used in the traditional CKF is listed as follows.

Time Update. Evaluate the cubature points $\widehat{\mathbf{x}}_{k-1}^{(i)}$.

$$
\begin{aligned}
& \widehat{\mathbf{x}}_{k-1}^{(i)}=\widehat{\mathbf{x}}_{k-1}^{+}+\sqrt{n \mathbf{P}_{k-1}^{+}} \xi_{i} \\
& \widehat{\mathbf{x}}_{k-1}^{(n+i)}=\widehat{\mathbf{x}}_{k-1}^{+}-\sqrt{n \mathbf{P}_{k-1}^{+}} \xi_{i}, \\
& \quad i=1,2, \ldots, n .
\end{aligned}
$$

Evaluate the propagated cubature points $\mathbf{X}_{k}^{(i)}$.

$$
\mathbf{X}_{k}^{(i)}=\mathbf{f}\left(\widehat{\mathbf{x}}_{k-1}^{(i)}\right) .
$$

Estimate the predicted state $\widehat{\mathbf{x}}_{k}^{-}$.

$$
\widehat{\mathbf{x}}_{k}^{-}=\frac{1}{2 n} \sum_{i=1}^{2 n} \mathbf{X}_{k}^{(i)} .
$$


Estimate the predicted error covariance $\mathbf{P}_{k}^{-}$.

$$
\mathbf{P}_{k}^{-}=\frac{1}{2 n} \sum_{i=1}^{2 n}\left(\mathbf{X}_{k}^{(i)}-\widehat{\mathbf{x}}_{k}^{-}\right)\left(\mathbf{X}_{k}^{(i)}-\widehat{\mathbf{x}}_{k}^{-}\right)^{\mathrm{T}}+\mathbf{Q}_{k-1} .
$$

Measurement Update. Evaluate the cubature points $\widehat{\mathbf{x}}_{k}^{(i)}$.

$$
\begin{aligned}
\widehat{\mathbf{x}}_{k}^{(i)} & =\widehat{\mathbf{x}}_{k}^{-}+\sqrt{n \mathbf{P}_{k}^{-}} \boldsymbol{\xi}_{i} . \\
\widehat{\mathbf{x}}_{k}^{(n+i)}=\widehat{\mathbf{x}}_{k}^{-}-\sqrt{n \mathbf{P}_{k}^{-}} \boldsymbol{\xi}_{i}, & \\
& \quad i=1,2, \ldots, n .
\end{aligned}
$$

Evaluate the propagated cubature points $\mathbf{Z}_{k}^{(i)}$.

$$
\mathbf{Z}_{k}^{(i)}=\mathbf{h}\left(\widehat{\mathbf{x}}_{k}^{(i)}\right)
$$

Estimate the predicted measurement $\widehat{\mathbf{z}}_{k}$.

$$
\widehat{\mathbf{z}}_{k}=\frac{1}{2 n} \sum_{i=1}^{2 n} \mathbf{Z}_{k}^{(i)}
$$

Estimate the measurement covariance matrix $\mathbf{P}_{z}$.

$$
\mathbf{P}_{z}=\frac{1}{2 n} \sum_{i=1}^{2 n}\left(\mathbf{Z}_{k}^{(i)}-\widehat{\mathbf{z}}_{k}\right)\left(\mathbf{Z}_{k}^{(i)}-\widehat{\mathbf{z}}_{k}\right)^{\mathrm{T}}+\mathbf{R}_{k} .
$$

Estimate the cross-covariance matrix $\mathbf{P}_{x z}$.

$$
\mathbf{P}_{x z}=\frac{1}{2 n} \sum_{i=1}^{2 n}\left(\widehat{\mathbf{x}}_{k}^{(i)}-\widehat{\mathbf{x}}_{k}^{-}\right)\left(\mathbf{Z}_{k}^{(i)}-\widehat{\mathbf{z}}_{k}\right)^{\mathrm{T}} .
$$

Estimate the Kalman gain $\mathbf{K}_{k}$.

$$
\mathbf{K}_{k}=\mathbf{P}_{x z} \mathbf{P}_{z}^{-1} .
$$

Estimate the updated state $\widehat{\mathbf{x}}_{k}^{+}$.

$$
\widehat{\mathbf{x}}_{k}^{+}=\widehat{\mathbf{x}}_{k}^{-}+\mathbf{K}_{k}\left(\mathbf{z}_{k}-\widehat{\mathbf{z}}_{k}\right) .
$$

Estimate the corresponding error covariance $\mathbf{P}_{k}^{+}$.

$$
\mathbf{P}_{k}^{+}=\mathbf{P}_{k}^{-}-\mathbf{K}_{k} \mathbf{P}_{z} \mathbf{K}_{k}^{\mathrm{T}} .
$$

From the algorithm we see that $2 n$ points are adopted when approximating the Gaussian pdf. To improve the filtering accuracy, more points, with corresponding weights, are needed.

\section{Fifth-Degree Cubature Rule and Cubature Filtering Algorithm}

3.1. Fifth-Degree Cubature Rule. The integral $I(\mathbf{f})$, for which it is difficult to find the analytical solution, can be approximated using the cubature rule $R(\mathbf{f})=\sum_{i=1}^{L} \omega_{i} \mathbf{f}\left(\mathbf{x}_{i}\right)$ by selecting the appropriate cubature points and corresponding weights, where $\mathbf{x}_{i}$ denotes the cubature points, $\omega_{i}$ denotes the weights that do not depend on the integrand, and $L$ denotes the number of cubature points. We will write $\mathbf{x}=\left(\begin{array}{llll}x_{1} & x_{2} & \cdots & x_{n}\end{array}\right)$ to denote an arbitrary point in real $n$-dimensional space. By a monomial of degree $d$, we mean a function of the form $x_{1}^{i_{1}} x_{2}^{i_{2}} \cdots x_{n}^{i_{n}}$, where the indices are nonnegative integers such that $i_{1}+i_{2}+\cdots+i_{n}=d$. The following definitions and lemma are introduced.

Definition 1 (see [21]). $S$ is a region in $n$-dimensional space; given $\mathbf{x} \in S$, the fully symmetric set of $\mathbf{x}, \sigma(\mathbf{x})$, is the set of all points $\left(\begin{array}{llll} \pm x_{j_{1}} & \pm x_{j_{2}} & \cdots & \pm x_{j_{n}}\end{array}\right)$, where $\left(\begin{array}{llll}j_{1} & j_{2} & \cdots & j_{n}\end{array}\right)$ is any permutation of $\left(\begin{array}{llll}1 & 2 & \cdots & n\end{array}\right)$.

Definition 2 (see [21]). A region $S$ is said to be fully symmetric if and only if $\mathbf{x} \in S$ implies $\sigma(\mathbf{x}) \subset S$.

Definition 3 (see [21]). An integration rule $R$ is said to be fully symmetric if and only if, whenever $\mathbf{x}$ is an abscissa of the rule $R$, every element of $\sigma(\mathbf{x})$ is an abscissa of $R$ and the same weight corresponds to all abscissas belonging to a given fully symmetric set.

Lemma 4 (see [21]). A fully symmetric rule R applied to a fully symmetric $n$-dimensional region $S$ is of degree $d$ if and only if it is exact for all monomials of degree $\leq d$ of the form

$$
x_{1}^{2 i_{1}} x_{2}^{2 i_{2}} \cdots x_{n}^{2 i_{n}}, \quad i_{1} \geq i_{2} \geq \cdots \geq i_{n}
$$

The following cubature rule is considered:

$$
\begin{aligned}
R(\mathbf{f})= & \omega_{1} \mathbf{f}\left(\begin{array}{lll}
0 & \cdots & 0
\end{array}\right)+\omega_{2} \sum_{\text {full sym }} \mathbf{f}\left(\begin{array}{lllll} 
\pm \lambda & 0 & \cdots & 0
\end{array}\right) \\
& +\omega_{3} \sum_{\text {full sym }} \mathbf{f}\left(\begin{array}{lllll} 
\pm \lambda & \pm \lambda & 0 & \cdots & 0
\end{array}\right)
\end{aligned}
$$

The above rule is fully symmetric; therefore, it will be of degree five if it is exact for the monomials $1, x_{1}^{2}, x_{1}^{4}$, and $x_{1}^{2} x_{2}^{2}$; thus the following equations are obtained:

$$
\begin{aligned}
I(1) & =\omega_{1}+2 n \omega_{2}+2 n(n-1) \omega_{3} \\
I\left(x_{1}^{2}\right) & =2 \lambda^{2} \omega_{2}+4(n-1) \lambda^{2} \omega_{3} \\
I\left(x_{1}^{4}\right) & =2 \lambda^{4} \omega_{2}+4(n-1) \lambda^{4} \omega_{3} \\
I\left(x_{1}^{2} x_{2}^{2}\right) & =4 \lambda^{4} \omega_{3} .
\end{aligned}
$$

For the case $I(\mathbf{f})=\int_{\mathrm{R}^{n}} \mathbf{f}(\mathbf{x}) e^{-\mathbf{x}^{\mathrm{T}} \mathbf{x}} d \mathbf{x}$, we have $I\left(x_{1}^{2 i_{1}} x_{2}^{2 i_{2}} \ldots\right.$ $\left.x_{n}^{2 i_{n}}\right)=\Gamma\left(i_{1}+1 / 2\right)\left(i_{2}+1 / 2\right) \cdots\left(i_{n}+1 / 2\right)$, where the Gamma function $\Gamma(z)$ satisfies $\Gamma(1 / 2)=\sqrt{\pi}$ and $\Gamma(z+1)=z \Gamma(z)$; thus 
we obtain

$$
\begin{aligned}
I(1) & =\underbrace{\Gamma\left(\frac{1}{2}\right) \cdots \Gamma\left(\frac{1}{2}\right)}_{n}=\pi^{n / 2} \\
I\left(x_{1}^{2}\right) & =\Gamma\left(1+\frac{1}{2}\right) \underbrace{\Gamma\left(\frac{1}{2}\right) \cdots \Gamma\left(\frac{1}{2}\right)}_{n-1}=\frac{1}{2} \pi^{n / 2} \\
I\left(x_{1}^{4}\right) & =\Gamma\left(2+\frac{1}{2}\right) \underbrace{\Gamma\left(\frac{1}{2}\right) \cdots \Gamma\left(\frac{1}{2}\right)}_{n-1}=\frac{3}{4} \pi^{n / 2} \\
I\left(x_{1}^{2} x_{2}^{2}\right) & =\Gamma\left(1+\frac{1}{2}\right) \Gamma\left(1+\frac{1}{2}\right) \Gamma \underbrace{\Gamma\left(\frac{1}{2}\right) \cdots \Gamma\left(\frac{1}{2}\right)}_{n-2} \\
& =\frac{1}{4} \pi^{n / 2} .
\end{aligned}
$$

Formula (21) is combined with formula (22) to solve the following parameters as

$$
\begin{aligned}
& \lambda^{2}=\frac{3}{2} \\
& \omega_{1}=\frac{n^{2}-7 n+18}{18} \pi^{n / 2} \\
& \omega_{2}=\frac{4-n}{18} \pi^{n / 2} \\
& \omega_{3}=\frac{1}{36} \pi^{n / 2} .
\end{aligned}
$$

Thus the specific form of rule $R(\mathbf{f})$ is achieved by substituting formula (23) into formula (20), and the total number of cubature points required for the rule is $1+2 C_{n}^{1}+4 C_{n}^{2}=2 n^{2}+1$. Furthermore, the integral $\int_{\mathrm{R}^{n}} \mathbf{f}(\mathbf{x}) e^{-\mathbf{x}^{\mathrm{T}} \mathbf{x}} d \mathbf{x}$ is written using the rule in the following form:

$$
\begin{aligned}
\int_{\mathbf{R}^{n}} \mathbf{f}(\mathbf{x}) e^{-\mathbf{x}^{\mathrm{T}} \mathbf{x}} d \mathbf{x} & \\
= & \frac{n^{2}-7 n+18}{18} \pi^{n / 2} \mathbf{f}\left(\begin{array}{lll}
0 & \cdots & 0
\end{array}\right) \\
& +\frac{4-n}{18} \pi^{n / 2} \sum_{\text {full sym }} \mathbf{f}\left(\begin{array}{llll}
\sqrt{\frac{3}{2}} & 0 & \cdots & 0
\end{array}\right) \\
& +\frac{1}{36} \pi^{n / 2} \sum_{\text {full sym }} \mathbf{f}\left(\begin{array}{lllll}
\sqrt{\frac{3}{2}} \sqrt{\frac{3}{2}} & 0 & \cdots & 0
\end{array}\right) .
\end{aligned}
$$

The following vectors are defined:

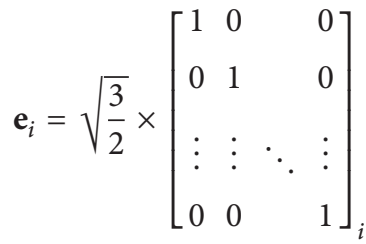

$$
\begin{aligned}
& \mathbf{p}_{i}^{+}=\mathbf{e}_{i}+\mathbf{e}_{j}, \quad i<j \\
& \mathbf{p}_{i}^{-}=\mathbf{e}_{i}-\mathbf{e}_{j}, \quad i<j
\end{aligned}
$$

$$
i, j=1,2, \ldots, n,
$$

where $[\cdot]_{i}$ denotes the $i$ th column of the matrix. From formula (5), the fifth-degree cubature rule approximating the Gaussian weighted integral of the nonlinear function is obtained by combining formula (24) with the vectors defined in formulae (25) as follows:

$$
\begin{gathered}
\int_{\mathbf{R}^{n}} \mathbf{f}(\mathbf{x}) N\left(\mathbf{x} ; \overline{\mathbf{x}}, \mathbf{P}_{x}\right) d \mathbf{x}=\frac{n^{2}-7 n+18}{18} \mathbf{f}(\overline{\mathbf{x}})+\frac{4-n}{18} \\
\cdot \sum_{i=1}^{n}\left[\mathbf{f}\left(\sqrt{2 \mathbf{P}_{x}} \mathbf{e}_{i}+\overline{\mathbf{x}}\right)+\mathbf{f}\left(-\sqrt{2 \mathbf{P}_{x}} \mathbf{e}_{i}+\overline{\mathbf{x}}\right)\right]+\frac{1}{36} \\
\cdot \sum_{i=1}^{n(n-1) / 2}\left[\mathbf{f}\left(\sqrt{2 \mathbf{P}_{x}} \mathbf{p}_{i}^{+}+\overline{\mathbf{x}}\right)+\mathbf{f}\left(-\sqrt{2 \mathbf{P}_{x}} \mathbf{p}_{i}^{+}+\overline{\mathbf{x}}\right)\right. \\
\left.+\mathbf{f}\left(\sqrt{2 \mathbf{P}_{x}} \mathbf{p}_{i}^{-}+\overline{\mathbf{x}}\right)+\mathbf{f}\left(-\sqrt{2 \mathbf{P}_{x}} \mathbf{p}_{i}^{-}+\overline{\mathbf{x}}\right)\right] .
\end{gathered}
$$

3.2. Fifth-Degree Cubature Kalman Filter. The following discrete nonlinear dynamic system is considered:

$$
\begin{aligned}
\mathbf{x}_{k} & =\mathbf{f}\left(\mathbf{x}_{k-1}\right)+\mathbf{w}_{k-1} \\
\mathbf{z}_{k} & =\mathbf{h}\left(\mathbf{x}_{k}\right)+\mathbf{v}_{k} \\
\mathbf{w}_{k} & \sim\left(0, \mathbf{Q}_{k}\right), \mathbf{v}_{k} \sim\left(0, \mathbf{R}_{k}\right),
\end{aligned}
$$

where $\mathbf{x}_{k} \in \mathbf{R}^{n_{x}}$ denotes the state vector, $\mathbf{z}_{k} \in \mathbf{R}^{n_{z}}$ denotes the measurement vector, $\mathbf{f}(\cdot)$ and $\mathbf{h}(\cdot)$ are known nonlinear functions, and the process noise $\mathbf{w}_{k}$ and measurement noise $\mathbf{v}_{k}$ are uncorrelated zero mean Gaussian white noise with covariance matrixes $\mathbf{Q}_{k}$ and $\mathbf{R}_{k}$, respectively.

The cubature points $\widehat{\mathbf{x}}$ and corresponding weights $\omega$ are obtained from the fifth-degree cubature rule shown in 
formula (26) as follows:

$$
\begin{aligned}
& \widehat{\mathbf{x}}_{1}=\overline{\mathbf{x}} \\
& \widehat{\mathbf{x}}_{1+i}=\overline{\mathbf{x}}+\sqrt{2 \mathbf{P}_{x}} \mathbf{e}_{i} \\
& \widehat{\mathbf{x}}_{n+1+i}=\overline{\mathbf{x}}-\sqrt{2 \mathbf{P}_{x}} \mathbf{e}_{i}, \\
& \widehat{\mathbf{x}}_{2 n+1+i}=\overline{\mathbf{x}}+\sqrt{2 \mathbf{P}_{x}} \mathbf{p}_{i}^{+} \\
& \widehat{\mathbf{x}}_{\left(n^{2}+3 n+2\right) / 2+i}=\overline{\mathbf{x}}-\sqrt{2 \mathbf{P}_{x}} \mathbf{p}_{i}^{+} \\
& \widehat{\mathbf{x}}_{n^{2}+n+1+i}=\overline{\mathbf{x}}+\sqrt{2 \mathbf{P}_{x}} \mathbf{p}_{i}^{-} \\
& \widehat{\mathbf{x}}_{\left(3 n^{2}+n+2\right) / 2+i}=\overline{\mathbf{x}}-\sqrt{2 \mathbf{P}_{x}} \mathbf{p}_{i}^{-}, \\
& \omega_{i}=\left\{\begin{array}{l}
\frac{n^{2}-7 n+18}{18}, \quad i=1 \\
\frac{1}{36}, \quad i=2,3, \ldots, 2 n+1
\end{array}\right.
\end{aligned}
$$

The proposed fifth-degree cubature Kalman filter is deduced by using the points and weights, shown, respectively, in formulae (28), under the Bayesian filtering framework with reference to the third-degree algorithm in [12], and the specific calculation steps are listed as follows.

Step 1 (filter initialisation).

$$
\begin{aligned}
& \widehat{\mathbf{x}}_{0}^{+}=E\left(\mathbf{x}_{0}\right) \\
& \mathbf{P}_{0}^{+}=E\left[\left(\mathbf{x}_{0}-\widehat{\mathbf{x}}_{0}^{+}\right)\left(\mathbf{x}_{0}-\widehat{\mathbf{x}}_{0}^{+}\right)^{\mathrm{T}}\right] .
\end{aligned}
$$

Cycle $k=1,2, \ldots$, and complete the following steps.

Step 2 (time update). Calculate the points $\widehat{\mathbf{x}}_{k-1}^{(i)}$ using the a posteriori state estimation $\widehat{\mathbf{x}}_{k-1}^{+}$and a posteriori error covariance matrix $\mathbf{P}_{k-1}^{+}$.

$$
\begin{aligned}
\widehat{\mathbf{x}}_{k-1}^{(1)} & =\widehat{\mathbf{x}}_{k-1}^{+} \\
\widehat{\mathbf{x}}_{k-1}^{(1+i)} & =\widehat{\mathbf{x}}_{k-1}^{+}+\sqrt{2 \mathbf{P}_{k-1}^{+}} \mathbf{e}_{i} \\
\widehat{\mathbf{x}}_{k-1}^{(n+1+i)} & =\widehat{\mathbf{x}}_{k-1}^{+}-\sqrt{2 \mathbf{P}_{k-1}^{+}} \mathbf{e}_{i},
\end{aligned}
$$$$
i=1,2, \ldots, n
$$

$$
\begin{array}{rl}
\widehat{\mathbf{x}}_{k-1}^{(2 n+1+i)} & =\widehat{\mathbf{x}}_{k-1}^{+}+\sqrt{2 \mathbf{P}_{k-1}^{+}} \mathbf{p}_{i}^{+} \\
\widehat{\mathbf{x}}_{k-1}^{\left(\left(n^{2}+3 n+2\right) / 2+i\right)} & =\widehat{\mathbf{x}}_{k-1}^{+}-\sqrt{2 \mathbf{P}_{k-1}^{+}} \mathbf{p}_{i}^{+} \\
\widehat{\mathbf{x}}_{k-1}^{\left(n^{2}+n+1+i\right)} & =\widehat{\mathbf{x}}_{k-1}^{+}+\sqrt{2 \mathbf{P}_{k-1}^{+}} \mathbf{p}_{i}^{-} \\
\widehat{\mathbf{x}}_{k-1}^{\left(\left(3 n^{2}+n+2\right) / 2+i\right)} & =\widehat{\mathbf{x}}_{k-1}^{+}-\sqrt{2 \mathbf{P}_{k-1}^{+}} \mathbf{p}_{i}^{-}, \\
i & i=1,2, \ldots, \frac{n(n-1)}{2} .
\end{array}
$$

Calculate the nonlinear propagation of $\widehat{\mathbf{x}}_{k-1}^{(i)}$ using $\mathbf{f}(\cdot)$.

$$
\mathbf{X}_{k}^{(i)}=\mathbf{f}\left(\widehat{\mathbf{x}}_{k-1}^{(i)}\right) \text {. }
$$

Calculate the a priori state estimation $\widehat{\mathbf{x}}_{k}^{-}$by weighted merging $\mathbf{X}_{k}^{(i)}$.

$$
\begin{aligned}
\widehat{\mathbf{x}}_{k}^{-}= & \frac{n^{2}-7 n+18}{18} \mathbf{X}_{k}^{(1)}+\sum_{i=2}^{2 n+1} \frac{4-n}{18} \mathbf{X}_{k}^{(i)} \\
& +\sum_{i=2 n+2}^{2 n^{2}+1} \frac{1}{36} \mathbf{X}_{k}^{(i)} .
\end{aligned}
$$

Calculate the a priori error covariance matrix $\mathbf{P}_{k}^{-}$.

$$
\begin{aligned}
\mathbf{P}_{k}^{-}= & \frac{n^{2}-7 n+18}{18}\left(\mathbf{X}_{k}^{(1)}-\widehat{\mathbf{x}}_{k}^{-}\right)\left(\mathbf{X}_{k}^{(1)}-\widehat{\mathbf{x}}_{k}^{-}\right)^{\mathrm{T}} \\
& +\sum_{i=2}^{2 n+1} \frac{4-n}{18}\left(\mathbf{X}_{k}^{(i)}-\widehat{\mathbf{x}}_{k}^{-}\right)\left(\mathbf{X}_{k}^{(i)}-\widehat{\mathbf{x}}_{k}^{-}\right)^{\mathrm{T}} \\
& +\sum_{i=2 n+2}^{2 n^{2}+1} \frac{1}{36}\left(\mathbf{X}_{k}^{(i)}-\widehat{\mathbf{x}}_{k}^{-}\right)\left(\mathbf{X}_{k}^{(i)}-\widehat{\mathbf{x}}_{k}^{-}\right)^{\mathrm{T}}+\mathbf{Q}_{k-1} .
\end{aligned}
$$

Step 3 (measurement update). Calculate the points $\widehat{\mathbf{x}}_{k}^{(i)}$ using the a priori state estimation $\widehat{\mathbf{x}}_{k}^{-}$and a priori error covariance matrix $\mathbf{P}_{k}^{-}$.

$$
\begin{aligned}
\widehat{\mathbf{x}}_{k}^{(1)} & =\widehat{\mathbf{x}}_{k}^{-} \\
\widehat{\mathbf{x}}_{k}^{(1+i)} & =\widehat{\mathbf{x}}_{k}^{-}+\sqrt{2 \mathbf{P}_{k}^{-}} \mathbf{e}_{i} \\
\widehat{\mathbf{x}}_{k}^{(n+1+i)} & =\widehat{\mathbf{x}}_{k}^{-}-\sqrt{2 \mathbf{P}_{k}^{-}} \mathbf{e}_{i}, \\
\widehat{\mathbf{x}}_{k}^{(2 n+1+i)} & =\widehat{\mathbf{x}}_{k}^{-}+\sqrt{2 \mathbf{P}_{k}^{-}} \mathbf{p}_{i}^{+} \\
\widehat{\mathbf{x}}_{k}^{\left(\left(n^{2}+3 n+2\right) / 2+i\right)} & =\widehat{\mathbf{x}}_{k}^{-}-\sqrt{2 \mathbf{P}_{k}^{-}} \mathbf{p}_{i}^{+} \\
\widehat{\mathbf{x}}_{k}^{\left(n^{2}+n+1+i\right)} & =\widehat{\mathbf{x}}_{k}^{-}+\sqrt{2 \mathbf{P}_{k}^{-}} \mathbf{p}_{i}^{-} \\
\widehat{\mathbf{x}}_{k}^{\left(\left(3 n^{2}+n+2\right) / 2+i\right)} & =\widehat{\mathbf{x}}_{k}^{-}-\sqrt{2 \mathbf{P}_{k}^{-}} \mathbf{p}_{i}^{-},
\end{aligned}
$$

$$
i=1,2, \ldots, \frac{n(n-1)}{2} .
$$


Calculate the nonlinear propagation of $\widehat{\mathbf{x}}_{k}^{(i)}$ using $\mathbf{h}(\cdot)$.

$$
\mathbf{Z}_{k}^{(i)}=\mathbf{h}\left(\widehat{\mathbf{x}}_{k}^{(i)}\right) .
$$

Calculate the predicted measurement value $\widehat{\mathbf{z}}_{k}$ by weighted merging $\mathbf{Z}_{k}^{(i)}$.

$$
\widehat{\mathbf{z}}_{k}=\frac{n^{2}-7 n+18}{18} \mathbf{Z}_{k}^{(1)}+\sum_{i=2}^{2 n+1} \frac{4-n}{18} \mathbf{Z}_{k}^{(i)}+\sum_{i=2 n+2}^{2 n^{2}+1} \frac{1}{36} \mathbf{Z}_{k}^{(i)}
$$
$\mathbf{P}_{z}$.

Calculate the predicted measurement covariance matrix

$$
\begin{aligned}
\mathbf{P}_{z}= & \frac{n^{2}-7 n+18}{18}\left(\mathbf{Z}_{k}^{(1)}-\widehat{\mathbf{z}}_{k}\right)\left(\mathbf{Z}_{k}^{(1)}-\widehat{\mathbf{z}}_{k}\right)^{\mathrm{T}} \\
& +\sum_{i=2}^{2 n+1} \frac{4-n}{18}\left(\mathbf{Z}_{k}^{(i)}-\widehat{\mathbf{z}}_{k}\right)\left(\mathbf{Z}_{k}^{(i)}-\widehat{\mathbf{z}}_{k}\right)^{\mathrm{T}} \\
& +\sum_{i=2 n+2}^{2 n^{2}+1} \frac{1}{36}\left(\mathbf{Z}_{k}^{(i)}-\widehat{\mathbf{z}}_{k}\right)\left(\mathbf{Z}_{k}^{(i)}-\widehat{\mathbf{z}}_{k}\right)^{\mathrm{T}}+\mathbf{R}_{k} .
\end{aligned}
$$

Calculate the cross-covariance matrix $\mathbf{P}_{x z}$.

$$
\begin{aligned}
\mathbf{P}_{x z}= & \frac{n^{2}-7 n+18}{18}\left(\widehat{\mathbf{x}}_{k}^{(i)}-\widehat{\mathbf{x}}_{k}^{-}\right)\left(\mathbf{Z}_{k}^{(i)}-\widehat{\mathbf{z}}_{k}\right)^{\mathrm{T}} \\
& +\sum_{i=2}^{2 n+1} \frac{4-n}{18}\left(\widehat{\mathbf{x}}_{k}^{(i)}-\widehat{\mathbf{x}}_{k}^{-}\right)\left(\mathbf{Z}_{k}^{(i)}-\widehat{\mathbf{z}}_{k}\right)^{\mathrm{T}} \\
& +\sum_{i=2 n+2}^{2 n^{2}+1} \frac{1}{36}\left(\widehat{\mathbf{x}}_{k}^{(i)}-\widehat{\mathbf{x}}_{k}^{-}\right)\left(\mathbf{Z}_{k}^{(i)}-\widehat{\mathbf{z}}_{k}\right)^{\mathrm{T}} .
\end{aligned}
$$

Calculate the Kalman filtering gain $\mathbf{K}_{k}$.

$$
\mathbf{K}_{k}=\mathbf{P}_{x z} \mathbf{P}_{z}^{-1} \text {. }
$$

Calculate the a posteriori state estimation $\widehat{\mathbf{x}}_{k}^{+}$.

$$
\widehat{\mathbf{x}}_{k}^{+}=\widehat{\mathbf{x}}_{k}^{-}+\mathbf{K}_{k}\left(\mathbf{z}_{k}-\widehat{\mathbf{z}}_{k}\right) .
$$

Calculate the a posteriori error covariance matrix $\mathbf{P}_{k}^{+}$.

$$
\mathbf{P}_{k}^{+}=\mathbf{P}_{k}^{-}-\mathbf{K}_{k} \mathbf{P}_{z} \mathbf{K}_{k}^{\mathrm{T}} .
$$

The pseudocode representing the proposed algorithm is given in Algorithm 1.

Remark 5. The proposed method is differential free; that is, there is no need to calculate the Jacobian matrix.

Remark 6. Compared with CKF of third degree, the filtering accuracy of the proposed method is improved to fifth degree.

Remark 7. The general method of computation of the cubature rule is given in the proposed filtering method, without dividing the intractable integral into spherical integral and radial integral.

\section{Mathematical Model for Orbit Determination}

4.1. Orbital Dynamics Model. Satellites in orbit are subjected to various perturbations, mainly including nonspherical gravitational perturbation, third body gravitational perturbation, atmospheric drag perturbation, and solar radiation pressure perturbation, among which the J2 nonspherical gravitational perturbation is the most influential perturbation. To describe the in-orbit motion of the satellite more accurately, the orbital dynamics model with $\mathrm{J} 2$ perturbation in the earth central fixed (ECF) coordinate system is used as follows to describe the orbit of the satellite [22]:

$$
\begin{aligned}
\dot{x}= & v_{x} \\
\dot{y}= & v_{y} \\
\dot{z}= & v_{z} \\
\dot{v}_{x}= & \omega_{e}^{2} x+2 \omega_{e} \cdot v_{y} \\
& +\frac{\mu x}{r^{3}}\left[J_{2}\left(\frac{R_{e}}{r}\right)\left(7.5 \frac{z^{2}}{r^{2}}-1.5\right)-1\right]+f_{x} \\
\dot{v}_{y}= & \omega_{e}^{2} y-2 \omega_{e} \cdot v_{x} \\
& +\frac{\mu y}{r^{3}}\left[J_{2}\left(\frac{R_{e}}{r}\right)\left(7.5 \frac{z^{2}}{r^{2}}-1.5\right)-1\right]+f_{y} \\
\dot{v}_{z}= & \frac{\mu z}{r^{3}}\left[J_{2}\left(\frac{R_{e}}{r}\right)\left(7.5 \frac{z^{2}}{r^{2}}-4.5\right)-1\right]+f_{z} \\
r= & \sqrt{x^{2}+y^{2}+z^{2}},
\end{aligned}
$$

where $\left(\begin{array}{llllll}x & y & z & v_{x} & v_{y} & v_{z}\end{array}\right)^{\mathrm{T}}$ denotes the position and velocity of satellite in ECF, $\mu$ denotes the earth gravity constant, $J_{2}$ denotes the harmonic coefficient, $R_{e}$ denotes the radius of the earth, $\omega_{e}$ denotes the angular velocity of the earth, and $\left(\begin{array}{lll}f_{x} & f_{y} & f_{z}\end{array}\right)^{\mathrm{T}}$ is the sum of other perturbations, which can be approximated as zero mean Gaussian white noise in the study. Formula (42) can be written in discrete state equation form as follows:

$$
\mathbf{x}_{k}=\mathbf{f}\left(\mathbf{x}_{k-1}\right)+\mathbf{w}_{k-1},
$$

where $\mathbf{x}_{k}=\left(\begin{array}{llllll}x_{k} & y_{k} & z_{k} & v_{x, k} & v_{y, k} & v_{z, k}\end{array}\right)^{\mathrm{T}}$ denotes the orbital state at time $k$ and $\mathbf{w}_{k}$ denotes the process noise.

4.2. Radar Measurement Model. The radar measurement model is described in local horizontal (LH) coordinate system, and the transformation matrix from ECF to $\mathrm{LH}$ is as follows:

$$
\mathbf{M}=\left[\begin{array}{ccc}
-\sin \lambda & \cos \lambda & 0 \\
-\sin \varphi \cos \lambda & -\sin \varphi \sin \lambda & \cos \varphi \\
\cos \varphi \cos \lambda & \cos \varphi \sin \lambda & \sin \varphi
\end{array}\right],
$$

where $\lambda$ and $\varphi$ denote the geocentric longitude and the geocentric latitude of radar, respectively, which can also be 
(1) Compute $\mathbf{e}_{i}=\sqrt{3 / 2} \times\left(\mathbf{I}_{n}\right)_{i}, \mathbf{p}_{i}^{+}=\mathbf{e}_{i}+\mathbf{e}_{j}, i<j$ and $\mathbf{p}_{i}^{-}=\mathbf{e}_{i}-\mathbf{e}_{j}, i<j$;

(2) Input: $\widehat{\mathbf{x}}_{0}^{+}, \mathbf{P}_{0}^{+}, \mathbf{z}_{k}$

(3) for $k=1$ to $z$ length do

(4) $\mathbf{S}_{k-1}^{+} \leftarrow \operatorname{chol}\left(\mathbf{P}_{k-1}^{+}\right)$;

(5) for $i=1$ to $2 n^{2}+1$ do

(6) Calculate $\widehat{\mathbf{x}}_{k-1}^{(i)}$ using $\widehat{\mathbf{x}}_{k-1}^{+}, \mathbf{S}_{k-1}^{+}, \mathbf{e}_{i}, \mathbf{p}_{i}^{+}$and $\mathbf{p}_{i}^{-}$;

(7) $\quad \mathbf{X}_{k}^{(i)} \leftarrow \mathbf{f}\left(\widehat{\mathbf{x}}_{k-1}^{(i)}\right)$;

(8) end

(9) $\widehat{\mathbf{x}}_{k}^{-} \leftarrow \sum_{i=1}^{2 n^{2}+1} \omega_{i} \mathbf{X}_{k}^{(i)}$;

(10) $\mathbf{P}_{k}^{-} \leftarrow \sum_{i=1}^{2 n^{2}+1} \omega_{i}\left(\mathbf{X}_{k}^{(i)}-\widehat{\mathbf{x}}_{k}^{-}\right)\left(\mathbf{X}_{k}^{(i)}-\widehat{\mathbf{x}}_{k}^{-}\right)^{\mathrm{T}}+\mathbf{Q}_{k-1}$;

(11) $\mathbf{S}_{k}^{-} \leftarrow \operatorname{chol}\left(\mathbf{P}_{k}^{-}\right)$;

(12) for $i=1$ to $2 n^{2}+1$ do

(13) Calculate $\widehat{\mathbf{x}}_{k}^{(i)}$ using $\widehat{\mathbf{x}}_{k}^{-}, \mathbf{S}_{k}^{-}, \mathbf{e}_{i}, \mathbf{p}_{i}^{+}$and $\mathbf{p}_{i}^{-}$;

(14) $\mathbf{Z}_{k}^{(i)} \leftarrow \mathbf{h}\left(\widehat{\mathbf{x}}_{k}^{(i)}\right)$;

(15) end

(16) $\widehat{\mathbf{z}}_{k} \leftarrow \sum_{i=1}^{2 n^{2}+1} \mathbf{Z}_{k}^{(i)}$;

(17) $\mathbf{P}_{z} \leftarrow \sum_{i=1}^{2 n^{2}+1} \omega_{i}\left(\mathbf{Z}_{k}^{(i)}-\widehat{\mathbf{z}}_{k}\right)\left(\mathbf{Z}_{k}^{(i)}-\widehat{\mathbf{z}}_{k}\right)^{\mathrm{T}}+\mathbf{R}_{k}$;

(18) $\mathbf{P}_{x z} \leftarrow \sum_{i=1}^{2 n^{2}+1} \omega_{i}\left(\widehat{\mathbf{x}}_{k}^{(i)}-\widehat{\mathbf{x}}_{k}^{-}\right)\left(\mathbf{Z}_{k}^{(i)}-\widehat{\mathbf{z}}_{k}\right)^{\mathrm{T}}$;

(19) $\mathbf{K}_{k} \leftarrow \mathbf{P}_{x z} \mathbf{P}_{z}^{-1}$;

(20) $\widehat{\mathbf{x}}_{k}^{+} \leftarrow \widehat{\mathbf{x}}_{k}^{-}+\mathbf{K}_{k}\left(\mathbf{z}_{k}-\widehat{\mathbf{z}}_{k}\right)$;

(21) $\mathbf{P}_{k}^{+} \leftarrow \mathbf{P}_{k}^{-}-\mathbf{K}_{k} \mathbf{P}_{z} \mathbf{K}_{k}^{\mathrm{T}}$;

(22) end

(23) Output: $\widehat{\mathbf{x}}_{k}^{+}, \mathbf{P}_{k}^{+}$

Algorithm 1: The pseudocode of the proposed algorithm. The fifth-degree cubature Kalman filter.

represented by geocentric coordinates $\left(\begin{array}{lll}x_{c} & y_{c} & z_{c}\end{array}\right)$. We define $\boldsymbol{\rho}=\left(\begin{array}{llllll}\rho_{x} & \rho_{y} & \rho_{z} & \dot{\rho}_{x} & \dot{\rho}_{y} & \dot{\rho}_{z}\end{array}\right)^{\mathrm{T}}$ as the satellite orbital states in $\mathrm{LH}$, and the following equations are obtained:

$$
\begin{aligned}
& {\left[\begin{array}{c}
\rho_{x} \\
\rho_{y} \\
\rho_{z}
\end{array}\right]=\mathbf{M} \cdot\left[\begin{array}{l}
x-x_{c} \\
y-y_{c} \\
z-z_{c}
\end{array}\right],} \\
& {\left[\begin{array}{c}
\dot{\rho}_{x} \\
\dot{\rho}_{y} \\
\dot{\rho}_{z}
\end{array}\right]=\mathbf{M} \cdot\left[\begin{array}{c}
v_{x} \\
v_{y} \\
v_{z}
\end{array}\right] .}
\end{aligned}
$$

The geometric relationship between orbital states and radar measurement values $(R \dot{R} A E)$ is obtained as follows:

$$
\begin{aligned}
& R=\sqrt{\rho_{x}^{2}+\rho_{y}^{2}+\rho_{z}^{2}} \\
& \dot{R}=\frac{\left(\rho_{x} \dot{\rho}_{x}+\rho_{y} \dot{\rho}_{y}+\rho_{z} \dot{\rho}_{z}\right)}{\sqrt{\rho_{x}^{2}+\rho_{y}^{2}+\rho_{z}^{2}}} \\
& A=\arctan \frac{\rho_{y}}{\rho_{x}} \\
& E=\arctan \frac{\rho_{z}}{\sqrt{\rho_{x}^{2}+\rho_{y}^{2}}},
\end{aligned}
$$

where $R$ denotes the ranging value, $\dot{R}$ denotes the velocity value, $A$ denotes the azimuth angle, and $E$ denotes the elevation angle. Formula (46) is written in the following measurement equation form:

$$
\mathbf{z}_{k}=\mathbf{h}\left(\mathbf{x}_{k}\right)+\mathbf{v}_{k},
$$

where $\mathbf{z}_{k}=\left(\begin{array}{llll}R_{k} & \dot{R}_{k} & A_{k} & E_{k}\end{array}\right)^{\mathrm{T}}$ denotes the measurement values at time $k$ and $\mathbf{v}_{k}$ denotes the measurement noise.

\section{The Numerical Experiment}

The simulation platform is built using the Satellite Tool Kit (STK) and MATLAB, the satellite runs in low-earth sunsynchronous orbit, and the reference orbit data is generated by the High-Precision Orbit Prediction (HPOP) algorithm. In HPOP, the 21-order earth gravity model is taken into account, the atmospheric drag coefficient $C_{d}=2.2$, the Jacchia-Roberts model is adopted as the atmospheric density model, the solar radiation pressure coefficient $C_{r}=1$, the area-mass ratio is $0.02 \mathrm{~m}^{2} / \mathrm{kg}$, the third body gravitational perturbations of sun and moon are considered, and the tidal perturbation is considered. The accuracy of ranging, velocity, and angle values is assumed to be $20 \mathrm{~m}, 0.1 \mathrm{~m} / \mathrm{s}$, and $0.015^{\circ}$, respectively. The six orbital elements, including semimajor axis (a), eccentricity (e), inclination ( $i)$, Right Ascension of Ascending Node (RAAN), argument of perigee $(\omega)$, true anomaly $(f)$, and the latitude and longitude of the radar station, are listed in Table 1. 


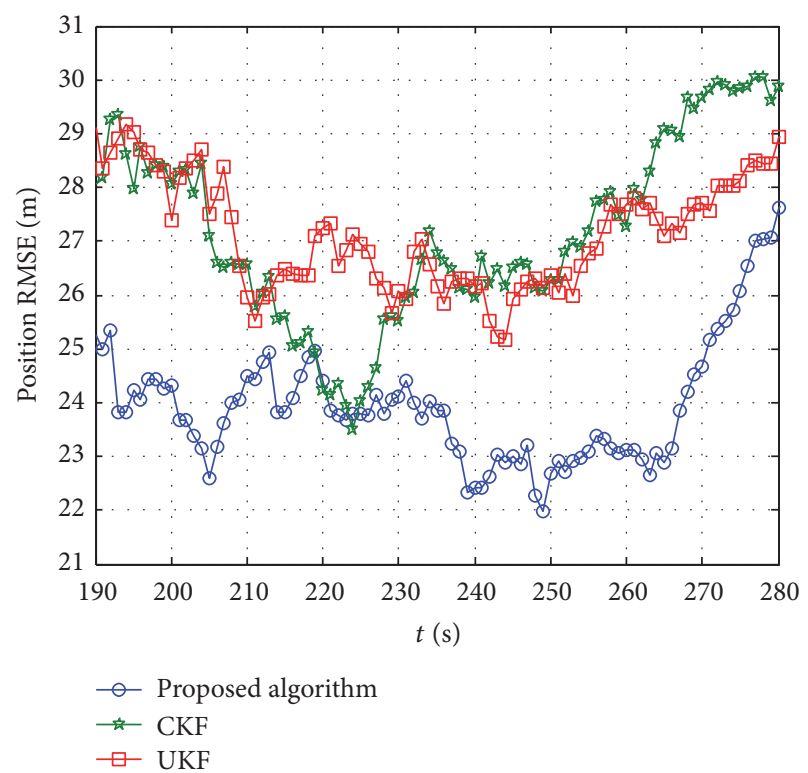

(a) Position RMSRs

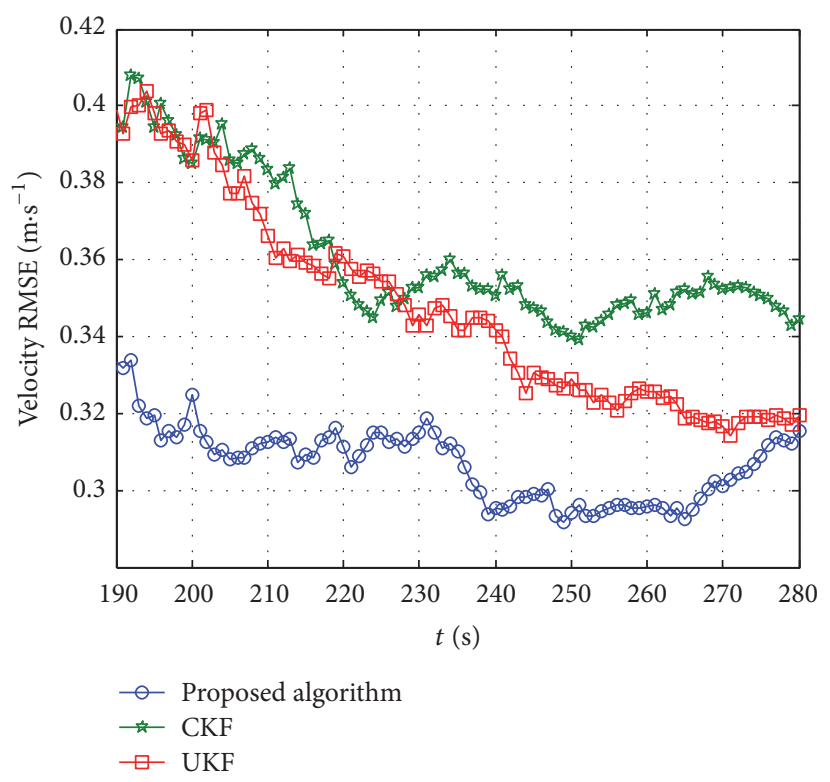

(b) Velocity RMSEs

FIGURE 1: Orbit determination RMSEs of three algorithms.

TABLE 1: The Six orbital elements and the latitude and longitude of the radar station.

\begin{tabular}{|c|c|c|c|c|c|c|c|c|}
\hline Epoch time/UTCG & $a / \mathrm{km}$ & $e$ & $i /^{\circ}$ & $\Omega /^{\circ}$ & $\omega /^{\circ}$ & $f 1^{\circ}$ & Longitude $^{\circ}$ & Latitude $/^{\circ}$ \\
\hline $1 \mathrm{Jul}, 2015,13: 05: 00$ & 6778.137 & 0 & 97.035 & 279.066 & 0 & 0 & 108.261 & 29.783 \\
\hline
\end{tabular}

TABLE 2: Average RMSE of three algorithms.

\begin{tabular}{lcc}
\hline Algorithm & Position RMSE $(\mathrm{m})$ & ${\text { Velocity RMSE }\left(\mathrm{m} \cdot \mathrm{s}^{-1}\right)}^{\mathrm{N}}$ \\
\hline UKF & 27.180 & 0.362 \\
CKF & 27.148 & 0.347 \\
Proposed algorithm & 23.944 & 0.307 \\
\hline
\end{tabular}

$\begin{array}{cccccc}\text { The } & \text { initial } & \text { filter } & \text { state } & \widehat{\mathbf{x}}_{0}^{+} & = \\ \left(\begin{array}{llllll}-2705129 & 5945598 & 1820143 & 2177 & -1252 & 7324\end{array}\right)^{\mathrm{T}} . \\ \begin{array}{cccccc}\text { The } & \text { initial } & \text { covariance } & \text { matrix } & \mathbf{P}_{0}^{+}\end{array}= \\ \operatorname{diag}\left(\begin{array}{llllll}10^{6} & 10^{6} & 10^{6} & 10^{2} & 10^{2} & 10^{2}\end{array}\right)\end{array}$

The access time from radar station to satellite is from 1 July, 2015, 16:14:00 to 1 July, 2015, 16:21:00, and the root mean square error (RMSE) is adopted to evaluate the realtime orbit determination results. The filtering cycle is $1 \mathrm{~s}$, and we ran 200 Monte-Carlo simulations. The UKF and CKF are compared in this experiment to validate the performance of the proposed algorithm. The RMSEs of the three algorithms are shown in Figure 1, and the statistical average RMSEs are summarised in Table 2. From the results it may be seen that the orbit determination accuracy obtained by UKF is almost consistent with that of CKF due to the two algorithms being made to adopt the third-degree deterministic sampling method. By contrast with CKF, the proposed fifth-degree cubature Kalman filter is capable of achieving a higher accuracy, and the position accuracy is increased by $3.204 \mathrm{~m}$ with velocity accuracy increased by $0.04 \mathrm{~m} / \mathrm{s}$. For a lowearth-orbit satellite, the atmospheric drag perturbation has an influence on the orbit, meaning that the state equation (formula (42)) cannot describe the orbit exactly, and if the high-precision orbit perturbation model is used, the excessive computation demand will impair the filtering algorithm's real-time performance; however, the errors caused by the orbital model are generally acceptable due to the access time of the LEO satellite being short.

\section{Conclusion}

In this paper, a novel fifth-degree cubature Kalman filter is proposed to improve the accuracy of real-time orbit determination by ground-based radar. The integral points and weights in the general cubature rule are solved by matching the monomials with degree not greater than five with their 
exact values, and then the fifth-degree cubature rule is deduced. The proposed novel fifth-degree cubature Kalman filter, which can achieve a higher filtering accuracy than UKF and CKF, is derived by using the aforementioned rule based on the Bayesian filtering framework. The simulation results show that the position accuracies achieved by CKF and the proposed algorithm are $27.148 \mathrm{~m}$ and $23.944 \mathrm{~m}$, respectively, with the velocity accuracies being $0.347 \mathrm{~m} / \mathrm{s}$ and $0.307 \mathrm{~m} / \mathrm{s}$, respectively. Compared with the results of CKF, the position accuracy and velocity accuracy are improved by $3.204 \mathrm{~m}$ and $0.04 \mathrm{~m} / \mathrm{s}$, respectively, thus verifying the validity of the proposed algorithm.

\section{Conflicts of Interest}

The authors declare that they have no conflicts of interest.

\section{Acknowledgments}

This work is supported partly by the National High Technology Research and Development Program of China (2015AA7026085).

\section{References}

[1] M. Sciotti, P. Besso, T. Flohrer, and H. Krag, "Low Earth Orbit objects tracking and orbit determination from ground-based phased array radar systems," in Proceedings of the International Radar Symposium, IRS 2011, pp. 591-596, Leipzig, Germany, 2011.

[2] X. Ning, C. M. Ye, J. Yang, and B. Shen, "Cubature kalman filtering for orbit determination of space targets," Chinese Journal of Radio Science, vol. 29, no. 1, pp. 27-32, 2014.

[3] "Cubature quadrature Kalman filter," IET Signal Processing, vol. 7, no. 7, pp. 533-541, 2013.

[4] J. Zarei and E. Shokri, "Convergence analysis of non-linear filtering based on cubature Kalman filter," IET Science, Measurement and Technology, vol. 9, no. 3, pp. 294-305, 2015.

[5] K. Reif, S. Gunther, E. Yaz, and R. Unbehauen, "Stochastic stability of the discrete-time extended Kalman filter," IEEE Transactions on Automatic and Control, vol. 44, no. 4, pp. 714-728, 1999.

[6] T. Ainscough, R. Zanetti, J. Christian, and P. D. Spanos, "Qmethod extended Kalman filter," Journal of Guidance, Control, and Dynamics, vol. 38, no. 4, pp. 752-760, 2015.

[7] C. D. Karlgaard and H. Shen, "Robust state estimation using desensitized divided difference filter," ISA Transactions, vol. 52, no. 5, pp. 629-637, 2013.

[8] M. F. Huber, "Chebyshev polynomial Kalman filter," Digital Signal Processing, vol. 23, no. 5, pp. 1620-1629, 2013.

[9] J. Sarmavuori and S. Sarkka, "Fourier-hermite kalman filter," IEEE Transactions on Automatic and Control, vol. 57, no. 6, pp. 1511-1515, 2012.

[10] S. Julier, J. Uhlmann, and H. F. Durrant-Whyte, "A new method for the nonlinear transformation of means and covariances in filters and estimators," Institute of Electrical and Electronics Engineers. Transactions on Automatic Control, vol. 45, no. 3, pp. 477-482, 2000.

[11] H. M. Menegaz, J. Y. Ishihara, G. A. Borges, and A. N. Vargas, "A systematization of the unscented Kalman filter theory,"
Institute of Electrical and Electronics Engineers. Transactions on Automatic Control, vol. 60, no. 10, pp. 2583-2598, 2015.

[12] I. Arasaratnam and S. Haykin, "Cubature Kalman filters," Institute of Electrical and Electronics Engineers. Transactions on Automatic Control, vol. 54, no. 6, pp. 1254-1269, 2009.

[13] I. Arasaratnam, S. Haykin, and T. R. Hurd, "Cubature Kalman filtering for continuous-discrete systems: theory and simulations," IEEE Transactions on Signal Processing, vol. 58, no. 10, pp. 4977-4993, 2010.

[14] J. Chen, N. Wang, L. Ma, and B. Xu, "Extended target probability hypothesis density filter based on cubature Kalman filter," IET Radar, Sonar and Navigation, vol. 9, no. 3, pp. 324-332, 2015.

[15] D. Potnuru, K. P. B. Chandra, I. Arasaratnam, D.-W. Gu, K. A. Mary, and S. B. Ch, "Derivative-free square-root cubature Kalman filter for non-linear brushless DC motors," IET Electric Power Applications, vol. 10, no. 5, pp. 419-429, 2016.

[16] L. Zhang, H. Yang, H. Lu, S. Zhang, H. Cai, and S. Qian, "Cubature Kalman filtering for relative spacecraft attitude and position estimation," Acta Astronautica, vol. 105, no. 1, pp. 254264, 2014.

[17] P. H. Leong, S. Arulampalam, T. A. Lamahewa, and T. D. Abhayapala, "A Gaussian-sum based cubature Kalman filter for bearings-only tracking," IEEE Transactions on Aerospace and Electronic Systems, vol. 49, no. 2, pp. 1161-1176, 2013.

[18] A. Noor, E. Serpedin, M. Nounou, and H. Nounou, "Reverse engineering sparse gene regulatory networks using cubature Kalman filter and compressed sensing," Advances in Bioinformatics, vol. 2013, Article ID 205763, 2013.

[19] B. Xu, Y. P. Xiao, W. Gao, Y. G. Zhang, Y. L. Liu, and Y. Liu, "Dual-model reverse CKF algorithm in cooperative navigation for USV," Mathematical Problems in Engineering, vol. 2014, Article ID 186785, 2014.

[20] L. Wang, M. Chen, G. Li, and Y. Fan, "Data-based control for humanoid robots using support vector regression, fuzzy logic, and cubature Kalman filter," Mathematical Problems in Engineering, Article ID 1984634, Art. ID 1984634, 19 pages, 2016.

[21] G. Phillips, "A survey of one-dimensional and multidimensional numerical integration," Computer Physics Communications, vol. 20, no. 1, pp. 17-27, 1980.

[22] X. L. Wang, S. Ding, and M. D. Su, "Application of UKF in geomagnetic field based autonomous navigation," Science of Surveying and Mapping, vol. 36, no. 6, pp. 103-105, 2011. 


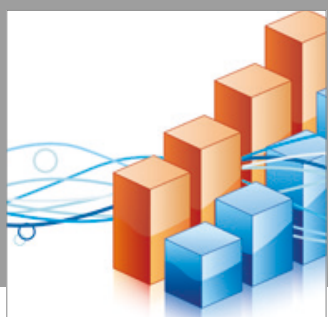

Advances in

Operations Research

vatersals

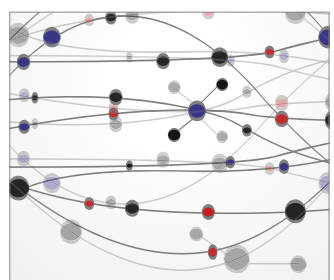

\section{The Scientific} World Journal
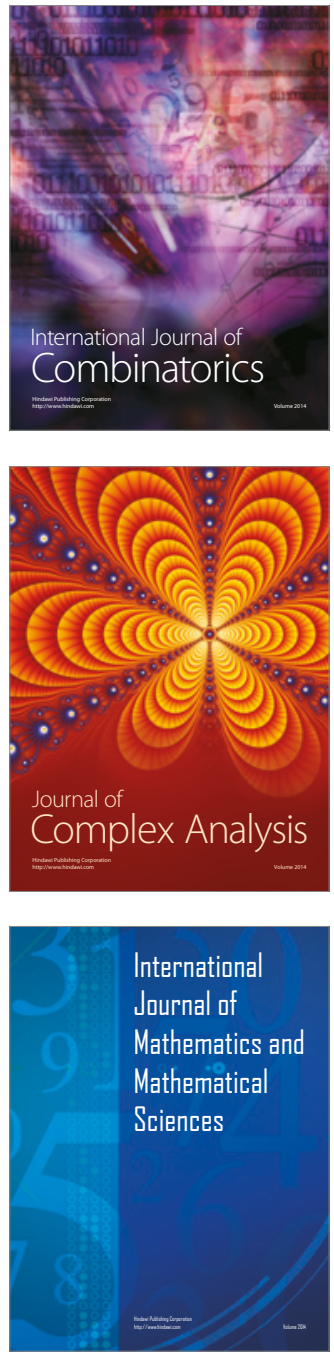
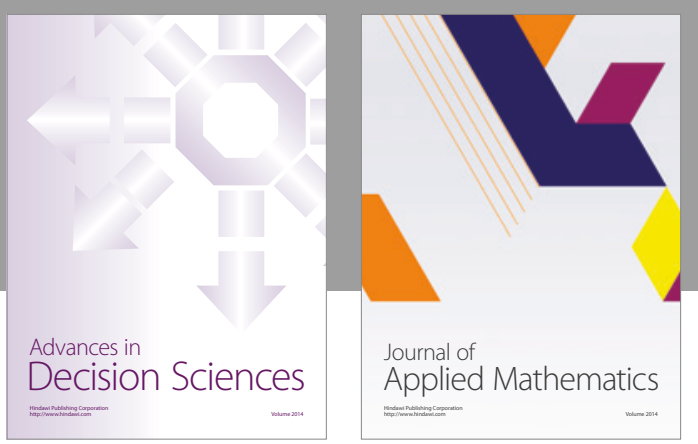

Algebra

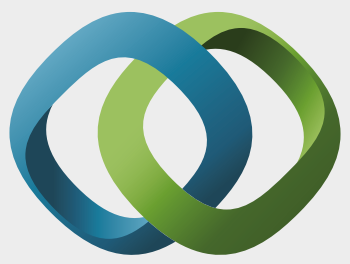

\section{Hindawi}

Submit your manuscripts at

https://www.hindawi.com
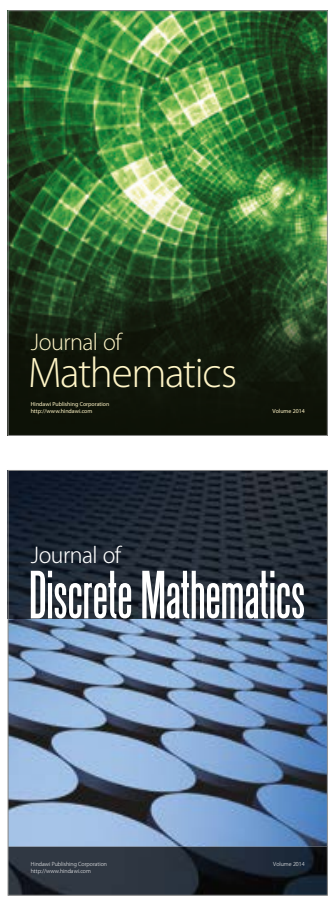

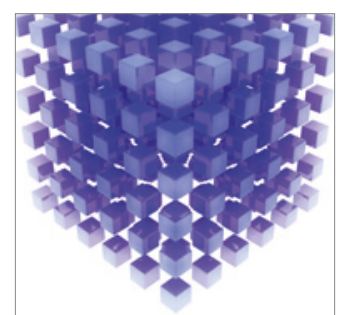

Mathematical Problems in Engineering
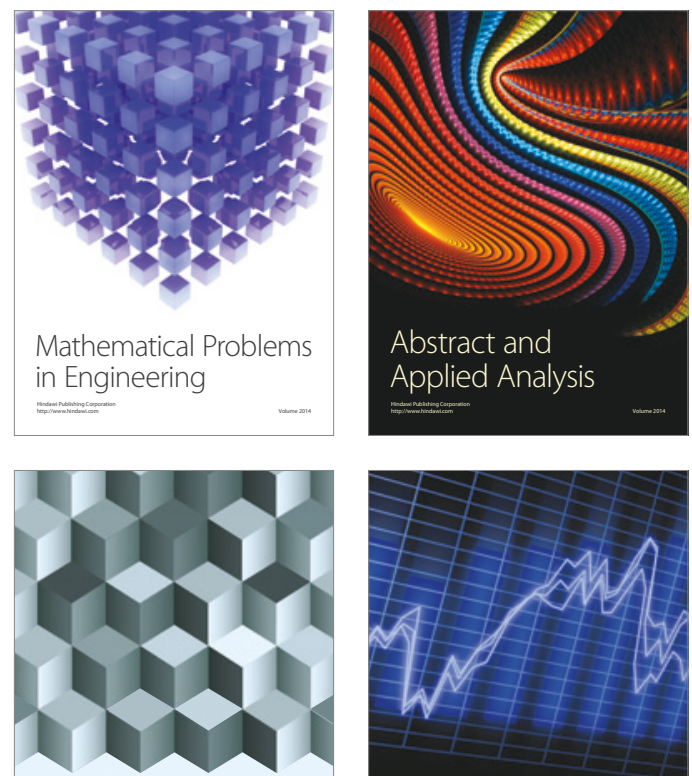

Journal of

Function Spaces

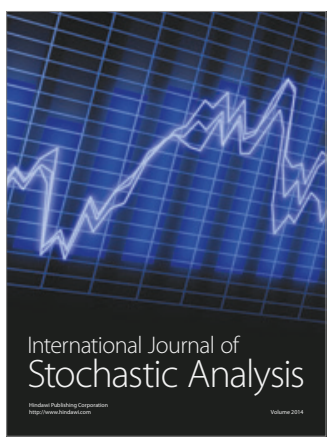

Probability and Statistics
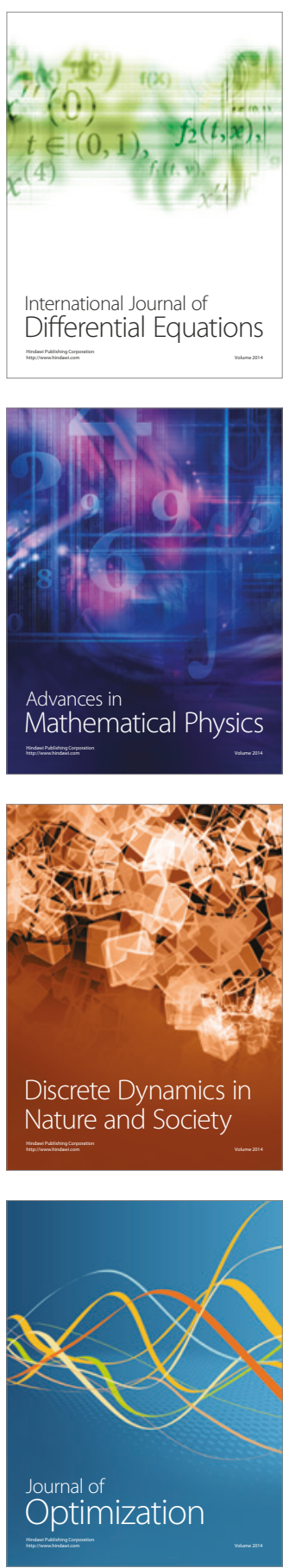